msh-mss Mathématiques et sciences humaines

183 | Automne 2008

Hommage à Georges-Th. Guilbaud

\title{
Ball. Trapp.
}

Ball. Trapp.

\section{Bernard Bru}

\section{(2) OpenEdition}

\section{Journals}

Édition électronique

URL : http://journals.openedition.org/msh/10803

DOI : $10.4000 / \mathrm{msh} .10803$

ISSN : 1950-6821

\section{Éditeur}

Centre d'analyse et de mathématique sociales de l'EHESS

\section{Édition imprimée}

Date de publication : 14 décembre 2008

Pagination : 55-70

ISSN : 0987-6936

\section{Référence électronique}

Bernard Bru, «Ball. Trapp. », Mathématiques et sciences humaines [En ligne], 183 | Automne 2008, mis en ligne le 15 décembre 2008, consulté le 23 juillet 2020. URL : http://journals.openedition.org/msh/ 10803 ; DOI : https://doi.org/10.4000/msh.10803 


\title{
BALL. TRAPP.
}

\author{
Bernard BRU ${ }^{1}$
}

Il y a une dizaine d'années, quelques-uns des nombreux amis de Guilbaud reçurent de lui des «fragments » d'un dossier intitulé «Ball. Trapp. », un ensemble de textes paginés, généralement tapés à la machine, certains longs d'une trentaine de pages, d'autres plus courts. Il ne s'agissait pas de l'amorce d'un traité sur le tir aux pigeons mais d'une recherche menée au cours de ses nombreux séjours dans des abbayes cisterciennes sur près d'un demi-siècle, ce qu'il appelait ses « Ballades trappistes », une sorte de journal de bord ou d'exploration, mais aussi des études de textes, enrichies au fil de ses lectures et de ses interrogations. Guilbaud précisait, à l'intention de correspondants, dont nous ignorons le nombre et l'identité : «J'écrivais beaucoup naguère - et publiais peu. Le très grand âge venant, l'idée m'est venue (saugrenue, j'avoue) de proposer de lire quelques pages (encore jamais lues) à quelques amis (proches ou lointains), non pour jugement, mais pour leur divertissement et, peut-être, aussi, l'occasion d'un petit moment de mémoire ». Il ajoutait que ces papiers contenaient toujours (un peu) de math., et "pour le reste de ma recherche, on peut y voir une sorte de braconnage. » Guilbaud voulait-il qu'on garde de lui aussi cette mémoire-là ? Peut-être, et, dans ce doute, en espérant ne pas le trahir, nous aimerions inviter les lecteurs de ce numéro spécial, à l'accompagner un court moment dans ses ballades cisterciennes, où il trouvait la paix de l'esprit, mais aussi, on le verra peut-être, certaines des idées et des intuitions qui structurent, animent, enrichissent son œuvre singulière et donnent à son enseignement cette limpidité et cette profondeur que chacun garde en mémoire.

\section{AD QUID VENISTI?}

D'abord un mot d'introduction pour le profane. Guilbaud, dont on sait l'extrême réserve sur tout ce qui le touchait personnellement, ne parlait pas volontiers de questions religieuses et encore moins de ses expériences monastiques. On sait bien sûr, et il ne s'en cachait pas, qu'il vécut ce qu'on appelle maintenant « le renouveau catholique des années $1930 »^{2}$, avec les avancées théologiques remarquables des Pères Jésuites de Lyon et des Dominicains du Saulchoir, de nouvelles perspectives d'action, syndicalisme

1 Université René Descartes, Laboratoire MAP5, 45 rue des Saints-Pères 75006 Paris, et Les Loges 01260 Le petit Abergement, bernard.bru@univ-paris5.fr

${ }^{2}$ On verra R. Rémond, «Le climat des années trente », Le Personnalisme d'Emmanuel Mounier, Paris, Seuil, 1985, p. 28, et P. Riché, Henri-Irénée Marrou, Paris, Cerf, 2003, p. 26-30. Ce renouveau sera freiné après la guerre, notamment par l'encyclique Humani generis de 1950, et par l'arrêt de l'expérience des prêtres-ouvriers en 1954. On verra à ce sujet, E. Fouilloux, Une église en quête de liberté, Paris, 1998. 
ouvrier et universitaire, mouvements d'action catholique, équipes sociales, etc., et des hommes de tout premier plan, religieux ou laïcs, que Guilbaud a bien connus et dont certains étaient de ses amis, Paul Couturier, Marcel Légaut, Paul Vignaux, Henri Marrou, Emmanuel Mounier, Pierre Chaillet, Louis-Joseph Lebret, Jacques Loew, Paul Archambault, tant d'autres, qui expliquent les engagements de Guilbaud au groupe œcuménique des Dombes, à Témoignage Chrétien ou à Économie et Humanisme, comme son orientation vers les applications des mathématiques à l'économie et aux sciences de l'homme, ou plutôt vers les correspondances (à double sens) entre mathématiques et sciences de l'homme. Mais il existe une autre piste, moins connue celle-là, quasi indépendante de ce «renouveau», qui joua peut-être dans la vie de Guilbaud, un rôle plus important encore, la piste cistercienne. Son origine remonte au début du $\mathrm{XII}^{\mathrm{e}}$ siècle, lorsque Bernard de Clarivaux et ses compagnons rejoignirent l'abbaye de Cîteaux, fondée en 1098 par Robert de Molesme, dans le but de revenir à une observance littérale de la Règle de saint Benoît. Cette nouvelle branche bénédictine (les «Cisterciens» ou les «Bernardins ») connut une expansion remarquable en Europe, plus de 300 abbayes, avec, pour certaines, des périodes de grande prospérité et de relâchement dans les observances, suivies parfois d'austérités plus grandes encore, à l'exemple de l'abbaye de Port-Royal, la plus connue d'entre elles. L'ordre cistercien fut une nouvelle fois réformé dans la seconde moitié du XVII ${ }^{\mathrm{e}}$ siècle par l'abbé de Rancé qui imposa à l'abbaye normande de la Trappe un retour aux observances les plus strictes de la Règle, suivi par d'autres abbayes de "Trappistes », avec des succès divers. Dissoutes à la Révolution, puis reconstituées au XIX ${ }^{\mathrm{e}}$ siècle, elles furent finalement organisées en congrégation autonome, l'ordre des cisterciens de la stricte observance (O.C.S.O.), par un décret du pape Léon XIII de 1892. L'ordre se développa beaucoup dans les années 1930 et après la Seconde guerre mondiale, jusqu'à compter plus de 4000 moines et 1800 moniales.

On sait assez bien quand Guilbaud découvrit l'ordre cistercien. Il le dit dans ses Ball. Trapp., en plusieurs endroits. C'était lors d'une « retraite » effectuée à l'abbaye cistercienne Notre-Dame des Dombes ${ }^{3}$, alors qu'il était élève du Lycée du Parc à Lyon, environ un an avant son entrée à l'École Normale, donc vers 1930-1931. Voilà ce qu'écrit Guilbaud, au sujet des retraites, dans une de ses «ballades », intitulée Ad quid venisti, p. 106 : «Il m'est arrivé, jadis, d'organiser des « retraites » - comme on disait qui rassemblaient une ou deux douzaines de personnes, et un orateur désigné (on disait : « prédicateur ») qui parlait au moins deux fois par jour.

J'avais noté qu'il était rare que ces « instructions » soient accordées au cadre, au lieu, à la liturgie.

\footnotetext{
${ }^{3}$ Fondée en 1863, l'abbaye des Dombes se trouve dans le département de l'Ain, au milieu des étangs de la Dombes, que les moines contribuèrent à assainir. Elle comptait entre les deux guerres une cinquantaine de moines. Elle a fermé en 2001, faute de recrutement. On verra, E. Goutagny, Cisterciens en Dombes, Paris, L'Harmattan, 2004.

Guilbaud fit plusieurs séjours aux Dombes. En particulier, il participa au groupe œecuménique des Dombes fondé en 1937 par l'abbé Paul Couturier, professeur de sciences au collège des Chartreux de Lyon, avec lequel Guilbaud s'était lié. On peut voir, M. Villain, L'abbé Paul Couturier, Paris, Casterman, 1957.

Signalons qu'à l'instar de beaucoup d'autres monastères, l'abbaye des Dombes fut un foyer de résistance pendant la guerre. Elle accueillit et aida des juifs, des résistants et les maquis de l'Ain. Ses actions de résistance lui valurent à la Libération d'être décorée de la légion d'honneur et de la croix de guerre à titre collectif, exemple unique ou peu s'en faut. On verra, E. Goutagny, Cisterciens dans les guerres, Paris, L'Harmattan, 2006.
} 
Je n'ai rencontré que le Père Albert Valensin pour s'en soucier, tout jésuite qu'il fût.

Il avait coutume d'interpréter à sa manière la Vigesima anotación qu'on peut lire dans les Ejercicios espirituales: Ignace de Loyola y note, sans trop insister, l'importance du lieu. Il dit le lien entre ce qu'il nomme apartamiento (séparation, mise à l'écart, on peut dire : retraite, si on veut) et la liturgie quotidienne...

Alors il nous emmenait aux Dombes, à Orval, à Aiguebelle. Il a dit sa préférence dans un commentaire de l'encyclique Mens nostra (publié dans la Nouvelle Revue Théologique, mars 1930) : dans ce texte (plus tard reproduit en volume) avant même de mentionner les «villas pieuses, gérées par les Pères de la Compagnie de Jésus », il cite comme lieux très recommandables pour pratiquer les Exercices spirituels «les hôtelleries de monastères cisterciens où la prière liturgique ajoute son recueillement à celui du cloître...».

C'était important, pensait-il, d'avoir présente à l'esprit la question : où sommes nous ? Et aussi : qui sont ceux qui nous accueillent ? Que font-ils ? Comment, pourquoi ?».

On peut sans doute déduire de ce texte que c'est lors d'une retraite prêchée par le Père Albert Valensin ${ }^{4}$, aux Dombes, que le jeune Guilbaud découvrit l'office cistercien. On sait même par un autre texte du même recueil qu'il s'agissait de l'Office de nuit, appelé aussi Vigiles, ou Matines, un dimanche, aux Dombes.

Essayons d'imaginer la scène. En 1930, l'office cistercien n'a pas encore été aménagé, ce qu'il sera à partir de 1955. Les Matines sont sonnées à 2 heures du matin. Depuis les étangs de la Dombes, un froid humide s'insinue partout. Les visiteurs sont accueillis à la tribune de l'église abbatiale, dans l'obscurité la plus complète. Un silence impressionnant, de ces silences qui parfois se font lorsque plus rien d'autre ne compte que la musique qui va se jouer ou la parole qu'on attend. Un silence qui est prière. Et soudain un chant, sans apprêt excessif, du grégorien sans ornement, à l'unisson d'un chœur de moines. Quelque chose d'improbable, de majestueux et de simple. Et l'office se poursuit suivi d'autres silences jusqu'à l'aube, avec l'office de Laudes, puis les autres offices du jour, jusqu'aux Complies, qui clôt la liturgie des heures.

Écoutons Guilbaud raconter un peu de cette expérience nocturne, dans un autre texte des Ball. Trapp., intitulé Te Deum Laudamus ${ }^{5}$. Peu avant la fin de la nuit, le dimanche, les moines chantent l'hymne du Te Deum (une composition du quatrième siècle dûe à l'évêque Nicetas de Ramesiana dans le Kosovo actuel). Il écrit :

J'étais donc ignorant, jusqu'à cette nuit où, pour la première fois, j'assistai à l'office cistercien (on disait matines, ou office de nuit). C'était un dimanche.

À la fin du troisième nocturne, juste avant la lecture de l'Évangile, j'entendis les moines chanter cet hymne (Te Deum Laudamus), et je pouvais suivre le texte dans le livre qu'on m'avait prêté.

\footnotetext{
4 «Tout jésuite qu'il fût», Albert Valensin était un véritable mystique, doué dès l'enfance du don d'oraison. Ami et correspondant de Maurice Blondel, Teilhard de Chardin, Paul Couturier, etc., il fut professeur de théologie aux Facultés catholiques de Lyon jusqu'en 1930. A cette date il démissionna et se consacra à l'encadrement de retraites pour les prêtres et les groupes d'étudiants, lyonnais en particulier, qu'il conduisait dans des couvents. On verra La vie intérieure d'un jésuite. Journal spirituel du P. Albert Valensin, 1873-1944, Paris, Aubier, 1953, p. 140. Le texte cité par Guilbaud est repris dans A. Valensin, Les Exercices spirituels : textes pontificaux annotés, Paris, Desclée de Brouwer, 1935.

${ }^{5}$ C'est aussi le titre d'un article publié par G.-Th. Guilbaud dans la revue Liturgie 87, 1993.
} 
Souvenir considérable, encore aujourd'hui ; non, ce n'est pas souvenir qu'il faudrait dire, car je n'ai guère d'images: je sais que ça a été le début de mon attachement à cette prière admirable. Je n'ai plus oublié, et mes rencontres successives se superposent et se mélangent dans ma mémoire.

Guilbaud, plus loin, dans le morceau Ad quid venisti, compare l'office monastique à la pratique de la montagne. On le comprend. La sortie du refuge, dans le froid et l'obscurité, la marche en silence, les hymnes des montagnes qui naissent de la nuit, le soleil du grand jour, le sommet, le retour dans la vallée, et fatigue et la joie de la fin du jour. Il écrit : « Montagne, Monastères, j'ai toujours associé les deux « sports », et aussi le troisième $\mathrm{M}$ : mathématiques ». Dans les trois cas, il s'agit d'une marche lente, parfois difficile, dans la nuit, le silence, qui prépare le corps et l'esprit à écouter et à chanter des hymnes d'action de grâce, des Te Deum. Un sport, une pratique, une action qui se transforment en contemplation mystique: une pratique mystique. Pour les Monastères, cela va de soi par définition. Admettons-le également pour la Montagne, métaphore classique. Pour les Mathématiques, la science positive par excellence, cela peut choquer certains bons esprits, mais il ne fait guère de doute que, pour Guilbaud au moins, cela ne faisait aucun doute, et il s'étonnait que son camarade Marcel Légaut, mathématicien de profession, paysan et mystique de cœur, aimât si peu les mathématiques. Mais «l'association» des trois $\mathrm{M}$ est personnelle et ne saurait être érigée en système général, sous peine d'amalgame ou de naïveté. Nous reprendrons ce point plus loin, s'il y a lieu.

Revenu à Lyon, puis monté à Paris après avoir été reçu au concours de l'ENS en 1932, Guilbaud nous dit qu'il tenta de comprendre mieux la nature de l'expérience hors du temps, mais bouleversante, vécue aux Dombes. Limitons-nous à un seul élément de preuve (dans un premier temps, nous en verrons d'autres plus loin). Dès son arrivée à Paris, Guilbaud suivit le cours inaugural que fit Etienne Gilson au Collège de France, où il avait été élu en 1932. Ce cours débuta le mardi 9 janvier 1933, salle 8 (nous précise Guilbaud), devant un public clairsemé. Gilson avait pris pour thème « la théologie mystique de saint Bernard », dont il fit un livre ${ }^{6}$, le premier sur ce qu'on ne considérait pas encore comme une théologie à part entière, mais plutôt comme une pratique mystique. Selon Gilson, certes, «Bernard ne fut aucunement métaphysicien, mais il devra aussi rester pour nous un théologien que sa puissance de synthèse et sa vigueur spéculative apparentent aux plus grands. Sans doute, sa théologie mystique est essentiellement la science d'une pratique, mais... C'est bien une science et il était difficile de pousser plus loin la rigueur de la synthèse ». Voilà une première initiation de Guilbaud aux sciences de l'homme, à l'une d'entre elles au moins, la science de la pratique mystique cistercienne, mais aussi par association, la science de la pratique mathématique, et pourquoi pas la science de la pratique montagnarde, si on arrivait à en discerner les principes et la langue, comme Gilson s'employa à le faire pour la langue et les principes de Bernard de Clairvaux. On pourrait imaginer l'existence chez Guilbaud d'un projet implicite englobant les trois $M$, dont ses innombrables textes épars sur tous les sujets imaginables seraient des indices ou des essais. Mais il serait hasardeux de développer ce thème sur lequel nous ne disposons d'aucun élément réel ou virtuel et qui, de toute façon, aurait bien fait rire Guilbaud, qui préférait parler, à ce sujet, de «braconnage ». Ajoutons néanmoins que Guilbaud écouta, l'année suivante, le second cours de Gilson au Collège de France, qui portait sur l'œuvre philosophique et théologique de Guillaume de Saint-Thierry, l'ami et le premier biographe de saint Bernard, un cours plus difficile, qui toutefois amena Guilbaud à se passionner pour

\footnotetext{
${ }^{6}$ E. Gilson, La théologie mystique de saint Bernard, Paris, J. Vrin, 1934, 1947, 1969, 1980, 1986. L'extrait cité figure dans la préface.
} 
Guillaume, maintenant mieux connu grâce aux textes édités à partir des années 1950 par les Sources chrétiennes et le Pain de Cîteaux, que Guilbaud suivit jusqu'à ces dernières années. Parallèlement à cette recherche de nature théorique, Guilbaud continua pendant plus de cinquante ans à faire des séjours, courts ou longs, dans des abbayes cisterciennes, d'abord à Port-du-Salut ${ }^{7}$, qu'il fréquenta beaucoup et bientôt partout en France et en Belgique. On se reportera à la carte qu'il avait jointe à ses Ball. Trapp., pour suivre ses pérégrinations cisterciennes «incognito », que nous reproduisons ici. Guilbaud écrit en commentaire (p. 109 de Ad quid venisti) :

Pas touriste, pas moine, alors: quoi? Chimère comme a dit un jour $S$. Bernard, mélange qui n'est ni l'un, ni l'autre, et caricature des deux.

«Mendiant et truand $»$ comme a dit Gerson ${ }^{8}$.

\footnotetext{
${ }^{7}$ L'abbaye de Port-du-Salut est la première abbaye de Trappistes rétablie sur le sol français après la Révolution. En 1815, des moines, venus de Darfeld en Allemagne, s'installèrent dans un ancien prieuré au bord de la Mayenne, non loin de Laval. L'abbaye, très importante dans les années 1930, est toujours en activité.
}

On ne sait pas de façon précise quand et comment Guilbaud a connu l'abbaye de Port-du-Salut. Les textes des Ball. Trapp. autorisent seulement des hypothèses. Guilbaud a participé dès 1932, avec son camarade Pierre Costabel, au « groupe tala » des étudiants catholiques de l'ENS, groupe qu'il a continué de fréquenter, plus épisodiquement, après 1935 et jusqu'en 1952. On sait aussi que, lorsqu'il était professeur de taupe, Guilbaud a longtemps fait partie de la Paroisse universitaire. On peut donc, sans grand risque, affirmer que c'est dans le cadre de l'un ou de l'autre de ces mouvements, qu'il a organisé des retraites dans des abbayes cisterciennes, et pourquoi pas à Port-du-Salut, assez proche de Paris. Sur la Paroisse universitaire dans les années 1930 et suivantes, on verra l'intéressante thèse de François Boirel, Catholiques en laïcité : l'exemple de la Paroisse universitaire. De Pierre Paris à Pierre Dabosville (1929-1963), Université Lyon 2, 1998. Guilbaud était très lié au Père Paris, (1884-1939), un prêtre de Saint-Sulpice, professeur au grand séminaire de Bordeaux, puis aumônier de la Paroisse universitaire. Sur le groupe tala des années trente, il ne semble pas exister d'étude particulière. Il faut se contenter d'allusions rapides dans des études plus générales ou de souvenirs publiés, d'ailleurs assez rares (par exemple, Paul Germain, Mémoire d'un scientifique chrétien, Paris, L’Harmattan, 2006). Sur les débuts du groupe tala, on se reportera à la thèse de Régis Ladous, Monsieur Portal et les siens, Paris, Cerf, 1985. En 1932, le groupe tala était encore marqué par la personnalité charismatique de Marcel Légaut, qui avait créé à l'ENS, dès 1923, une cellule de prière suivant une règle quasi monastique. Le groupe semble ensuite avoir éclaté, Marcel Légaut créant un groupe tala parallèle à l'ENS de Saint-Cloud. On verra les souvenirs de Légaut dans, B. Feillet, Marcel Légaut. Patience et passion d'un croyant, interviews, Paris, Centurion, 1976. De 1929 à 1944, le groupe tala eut pour «aumônier » le père Gaston Brillet (18781966), un oratorien discret et cultivé, supérieur général de son ordre de 1929 à 1949, qui eut une influence importante sur la vie du groupe, mais nous n'avons pas retrouvé son nom dans les Ball. Trapp. Sur sa vie, on verra le numéro spécial de la revue Oratoriana, 13 (1967), qui lui est consacré, en particulier les pages 92 à 99 qui concernent son action rue d'Ulm. Le groupe tala se réunissait en général le vendredi pour la messe dans la chapelle des Pères du Saint-Esprit, rue Lhomond. Il organisait également des conférences hebdomadaires sur des sujets généraux et des retraites deux ou trois fois par an. On verra aussi, P. Golliet, Jean Badelle (1917-1941), sa vie et sa pensée, Clermont-Ferrand, Laboureur et Cie, 1944. Ce livre permet de cerner quelque peu la personnalité d'un des responsables les plus remarquables du groupe tala, Jean Badelle, et à travers lui, celle de quelques-uns des membres du groupe, souvent issus de familles provinciales très croyantes, militants de la (toute nouvelle) J.E.C. dans leurs lycées respectifs, et, depuis la condamnation de 1926, aussi loin que possible de la mouvance monarchiste de l'Action Française, pourtant active et assez bien représentée à l'ENS. Il n'est d'ailleurs pas facile non plus de suivre le groupe Action Française de l'ENS, qui ne fréquentait pas le groupe tala, jugé moderniste, voire crypto-socialiste. Robert Brasillach, qui fut délégué de l'Action Française rue d'Ulm, et se convertit très tôt au fascisme mussolinien puis hitlérien, n'est que peu représentatif du groupe AF de l'ENS, provincial, antiallemand, antisémite et traditionnaliste, auquel Guilbaud n'adhéra en aucune façon. Sur les rapports entre catholiques et AF, on verra, J. Prévotat, Les catholiques et l'Action française: histoire d'une condamnation, 1899-1939, Paris, Fayard, 2001.

${ }^{8}$ Guilbaud cite en plusieurs endroits de ses Ball. Trapp. (notamment dans 153, paragraphe suivant), des textes de Jean Gerson de la fin du XIV ${ }^{\mathrm{e}}$ siècle, écrits en français, présentés par Pierre Pascal, et réunis sous le titre, Initiation à la vie spirituelle, Paris, Gallimard, 1943. L'un de ces textes s'intitule «La mendicité spirituelle », et commence ainsi, p. 112 : «Tu qui n'as rien et par ton labeur ne sais et ne peux 
Pèlerin, peregrinus, passant, étranger.

Tu loges à l'hôtellerie, mais tu n'es pas un «client». Ne pas confondre. Si tu reviens plusieurs fois, tu finiras par être connu. Tâche de ne pas devenir un " habitué ». L'incognito serait préférable. Mais on ne peut tout de même pas changer d'abbaye à chaque fois. Depuis le temps, j'en ai pratiqué une douzaine - très inégalement d'ailleurs - cela fait plus de la moitié des possibles (France et Belgique). Il sera sage de s'en tenir là.

À chacun sa route.

Avant de clore ce paragraphe introductif et de suivre rapidement une ou deux ballades guibaldiennes, revenons sur son titre, Ad quid venisti, un «slogan» de Guilbaud, après avoir été celui de Bernard et de ses continuateurs.

Tout commence par une citation. Guillaume de Saint-Thierry, dans sa biographie première de son ami Bernard (qui mourra cinq ans après lui), écrit que l'abbé de Clairvaux gardait dans son cœur et disait souvent Ad quid venisti ${ }^{9}$. Comment lire cette citation ? Sans doute, pour Bernard et les moines de Cîteaux était-ce une allusion à la Règle de saint Benoît, au chapitre 60 , où il est question des prêtres qui se présentent dans les monastères et demandent à y être admis de droit. La Règle dit qu'on se gardera bien d'y consentir et qu'on devra d'abord leur demander, comme dans l'Évangile de Matthieu 26, 50 : «Amice, ad quid venisti », ce que le traducteur bénédictin de la Règle transforme en «Ami, qu'êtes vous venu faire ici ?» ${ }^{10}$. Et il semble que ce soit en ce premier sens que Guilbaud ait pris la citation de Guillaume. Pourquoi es tu là ? Une question que peut se poser un retraitant, ou un moine, enfermé pour quelques jours, ou pour toujours, dans un monastère hors du monde. Une phrase que tout homme peut se poser lorsqu'il se trouve, par suite de circonstances diverses, transporté hors de luimême ou de ce qu'il avait pris l'habitude de considérer comme lui-même.

Mais les choses sont moins simples qu'il y parait. On se trouve en effet, nous dit Guilbaud, dans le cas d'une de ces fameuses « chaînes de citations » ou « d'emprunts à l'Écriture Sainte, avec chaque fois un contexte différent, et glissement de sens », fréquents dans la « littérature médiévale ». D'où est-on parti ? Peut-on seulement saisir le sens initial de ces trois mots ? Il faut revenir au texte de l'Évangile de Matthieu, cité

quelconque chose acquérir, crois mon conseil : apprends le métier de mendier et truander, et que ton pourchas te soit en lieu de rente. Pourquoi mourrais-tu de faim, de soif et de froidure ? A blâmer serait une telle honte ou paresse ». (pourchas vient de pourchasser : chercher sa vie).

Pierre Pascal (1890-1983), normalien, slaviste, fut professeur à la Sorbonne. Membre de la mission militaire française en Russie en 1916, et passionné par la révolution bolchevique, il resta en URSS jusqu'en 1933, où il rédigea sa célèbre thèse sur les débuts du Raskol et des vieux-croyants dans l'Église orthodoxe russe, et fut l'un des collaborateurs de Lénine.

Pour acheter un tel volume en 1943, il fallait être lié à Pierre Pascal d'une façon ou d'une autre, ou (et) poursuivre une recherche sur la « vie spirituelle », sans que nous en sachions davantage.

${ }^{9}$ Guilbaud cite le texte latin de Guillaume : Ad custodiam sui cordis hoc semper in corde saepe etiam in ore habebat : ad quid venisti », Vita prima Bernardi, traduction française de François Guizot, Collection des mémoires relatifs à l'histoire de France, ..., Paris, J. L. J. Brière, libraire, 1825, chapitre IV, p. 171, sur Gallica, réédité dans, Vie de Saint Bernard de Clairvaux, Clermont-Ferrand, Paléo, 2004.

Guizot traduit : pensant plutôt à la garde de son cœur et à la constance de son dessein, « il avait souvent dans l'esprit et même dans la bouche: 'Bernard, Bernard, à quoi en es-tu venu ?' ». Une version « juste milieu » du Ad quid venisti.

Guilbaud a ajouté une note érudite à cet endroit : «On trouve dans l'édition Mabillon des œuvres de S. Bernard, un opuscule: Opusculum in haec verba Ad quid venisti. Ce n'est pas un écrit de Bernard, c'est une compilation d'exhortations pieuses et banales, à l'intention des novices. Ce texte nous montre seulement que le proverbe Ad quid venisti est resté longtemps en usage».

${ }^{10}$ La Règle de saint Benoît, traduction française, Saint Benoît de Port Valais, Le Bouveret, 1961, p. 169, qui cite en note Mt, XXVI, 50. 
par la Règle : Amice, ad quid venisti. Il s'agit d'une phrase que le Christ adresse à Judas, au moment où ce dernier s'apprête à le désigner aux hommes chargés de l'arrêter. Il n'y a pas de point d'interrogation, ni d'exclamation, inconnus des langues anciennes. On peut donc traduire la phrase également comme une affirmation, une interrogation ou une exclamation, ce que les traducteurs ont fait de mille façons que Guilbaud collectionnait et dont nous donnons un échantillon au hasard :

- ami voilà donc pourquoi tu es là (Bible Bayard, 2001)

- ainsi te voilà ! (Pléiade, 1971)

- mon ami, qu'êtes vous venu faire ici ? (Le maître de Sacy)

- tu es venu dans ce but mon ami (Amphoux)

- compagnon, c'est pour cela que tu es là (Chouraqui)

- ami, ce que tu es venu faire, fais le (Segond)

- ce que tu es venu faire, fais le vite (Alliance biblique universelle)

- fais ta besogne (Bible de Jérusalem)

- etc.

Littéralement le latin, ad quid venisti, pourrait se traduire «ce pour quoi tu es venu », suivant le grec original, qui lui se traduit mot à mot « à cause de ce pour quoi tu es là ». Mais cela n'améliore guère la situation.

Le sens originel moyen, suivant les traducteurs, serait donc une affirmation. Le Christ et Judas savent tous deux ce qui va se passer, et en conviennent, avec cependant une importante variabilité, qui garde au sens toute sa richesse d'incertitude, de doute, de potentiel, de «glissement» suivant les contextes et les auteurs. Ce qui peut expliquer le faux sens volontaire, la forme interrogative et insistante de la Règle de saint Benoît et de la tradition cistercienne : que viens-tu faire ici ?

Faut-il aller plus loin? «À quoi pourrait mener cette recherche érudite ? écrit Guilbaud, sinon à ceci : ces trois mots ont résonné en l'esprit de nombreux ascètes au cours des siècles - comme un rappel permanent à la conversion, rappel coloré par la crainte d'y manquer. Sans vergogne, ces trois mots, je me les suis appropriés, à mon tour. J'en fais un mot d'ordre, un mot de passe. Ad quid venisti ? C'est une question. Qui parle ? J'imagine d'abord, j'entends déjà, des amis, des camarades, des collègues, surpris, s'étonnant, ne comprenant pas : «Qu'est ce que tu fous ici ?» diraient-ils. Ou en latin : Ad quid venisti ?»

\section{153}

Le fragment le plus long des Ball.Trapp., que nous ayons pu consulter, s'intitule 153. Il s'agit d'un texte difficile à lire, écrit par Guilbaud sans doute il y a une quinzaine d'année, une sorte de pérégrination à travers ses lectures « cisterciennes » et d'évocation d'amis très chers. Il débute ainsi : «Il y a bien longtemps, j'étais jeune, je cherchais à comprendre ce que pouvait être «la vie spirituelle»- comme on disait chez les chrétiens. Ce ne fut donc pas tout à fait par hasard que je trouvai chez un libraire une petite brochure (tiré à part de la Revue dominicaine La vie spirituelle) intitulée : Les grands mystiques: Saint Nil, Traité de la prière, traduction française par Dom Jean 
Joliet, moine de Solesme, juillet 1925, 30 pages $»{ }^{11}$. Cela devait se passer en 1932 ou en 1933. Guilbaud nous dit que l'impression qu'il en tira fut "considérable », au point qu'il commença à recopier le texte original dans le tome 79 de la Patrologie grecque ${ }^{12}$ et qu'il tenta de le traduire pour son compte. Au hasard de ses visites aux libraires, il se procura d'autres traductions du Traité de la prière de saint Nil, restitué finalement à Evagre, son auteur présumé, un père du désert de Nitrie du IV ${ }^{\mathrm{e}}$ siècle. Le fragment 153 est consacré en partie à un commentaire libre du Traité, et à des développements qui exploitent notamment un principe original de «correspondance » de deux pratiques chères à Guilbaud, l'arithmétique et la prière, en prenant en compte des textes échelonnés sur deux ou trois mille ans d'histoire approximativement.

Le texte étant assez long, nous n'en extrayons qu'une ou deux pages, choisies (pas tout à fait) au hasard. D'abord les premières pages «B à E», dans lequel Guilbaud nous dit que c'est le thème du Traité, la prière, qui l'a d'abord attiré, précisément ce qu'il cherchait. Mais il existe d'innombrables traités sur la prière, de toutes sortes, de toutes les époques, dans toutes les religions, jusqu'aux analyses universitaires de stricte observance, sociologiques de la thèse de Mauss, psychologiques de la thèse de Segond, philosophiques de Brémond, etc. Toute une vie n'y suffirait pas, et ce n'est pas le but recherché par le jeune Guilbaud, alors âgé de 20 ans, étudiant de mathématiques à l'ENS. Ce qui l'a décidé à poursuivre, c'est le prologue, dans lequel l'auteur du $\mathrm{IV}^{\mathrm{e}}$ siècle «manifeste un certain goût pour l'Arithmétique, or j'aime l'arithmétique ». Guilbaud commence par rappeler, ce qui est bien connu, que le Traité d'Evagre est fractionné en petits articles ou chapitres. «Le nombre des articles a été choisi pour signifier quelque chose : cent cinquante trois ». Évidemment à cause des 153 gros poissons de la dernière "pêche miraculeuse » (Jean 21, 11), comme le souligne le prologue : «En divisant mon traité en 153, je t'envoie un ravitaillement évangélique ». Mais, pour Evagre, 153 signifie aussi quelque chose en tant que nombre, c'est un nombre «triangulaire », non pas comme le croient certains commentateurs, parce qu'il est composé de trois chiffres, mais parce que $153=1+2+3+\ldots \ldots \ldots+17$, et que des cailloux rangés verticalement suivant cette progression dessinent un triangle, ce qui, ajoute Guilbaud, «était classique depuis l'Antiquité, Théon, Proclus, Boèce, jusqu'au Traité $d u$ Triangle Arithmétique de Blaise Pascal (1654)». Suivons maintenant Guilbaud dans son développement :

\footnotetext{
${ }^{11}$ Il s'agit en réalité de Jehan Joliet, sur lequel on verra la thèse de H.-P. Delcourt, Dom Jehan Joliet (1870-1937). Un projet de monachisme bénédictin chinois, Paris, Cerf, 1988.

Saint Nil d'Ancyre est un moine grec du IVe siècle du couvent du mont Sinaï. Plus loin dans le texte, Guilbaud nous apprend que, vers 1939, il a acheté un tiré à part du Père Irénée Hausherr s.j., («Le Traité de l'Oraison d'Evagre le Pontique (pseudo-Nil) », Revue d'ascétique et de mystique 15, 1934, p. 34-96, 113-170), donnant une nouvelle traduction du Traité, et démontrant qu'en réalité le Traité de la prière n'est pas de saint Nil mais d'Evagre du Pont, un moine du IV ${ }^{\mathrm{e}}$ siècle également, qui vécut en Egypte sur le site monastique des «cellules» de Nitrie, et qui est l'auteur d'une œuvre importante. Cette attribution semble admise par tout le monde maintenant. L'article de I. Hausherr est repris dans Les Leçons d'un contemplatif, le traité de l'oraison d'Evagre le Pontique, Paris, Beauchesne, 1960. Depuis, le Traité d'Evagre a été réédité à plusieurs reprises ; Guilbaud nous dit qu'il s'est procuré les traductions contenues dans la Philocalie des Pères neptiques ..., vol. 8, Bégrolles-en-Mauges, Abbaye de Bellefontaine, 1987 (traduction de Lucien Renault), et dans De la prière à la perfection / Evagre le Pontique, Paris, Migne, diff. Brépols, Collection Les Pères de la Foi 47, 1992, (traduction Marie-Odile Goudet). Signalons enfin que Guilbaud, tout en saluant l'érudition patristique du père Hausherr, indique très nettement que celui-ci n'a rien compris à l'arithmétique d'Evagre. Sur ce dernier, on verra A. Guillaumont, Un philosophe au désert : Evagre le Pontique, Paris, J. Vrin, 2004.

${ }^{12}$ La Patrologie grecque, éditée par J. P. Migne, à Montrouge, entre 1857 et 1866, comporte 161 volumes in-quarto. Elle regroupe les œuvres des pères grecs jusqu'en 1478. On la trouve en support électronique aux éditions Champion électronique.
} 
Bien sûr je comprenais qu'Evagre avait fragmenté son enseignement en 153 morceaux (il n'est pas le seul paraît-il) "à cause »du dernier chapitre du quatrième évangile, et pour nous y faire penser. Mais la lettre-prologue me disait quelque chose de plus que je me résumais ainsi, brutalement: l'arithmétique, c'est comme la prière. Voyons cela d'un peu plus près. "Toutes choses vont par paires", dit-il d'abord, citant le proverbe du Siracide ${ }^{13}$, et poursuit : c'est ainsi qu'il y a une double "manière » pour la prière mais aussi pour le nombre. Les deux côtés de la prière sont traditionnellement connus : actif, contemplatif. (Joliet disait: pratique, spéculatif, ce que Hausherr lui reproche, allez savoir pourquoi).

Pour un lecteur d'aujourd'hui ces deux termes sont un peu usés, mais il espère que la suite du texte l'instruise, puisqu'on y traite de la prière, tout au long des 153 chapitres.

Pour ce qui est du Nombre, je dois me contenter du prologue; on y trouve quelques indications, parcimonieuses, il est vrai.

Le nombre, lui aussi, a deux côtés. L'une des quatre traductions disponibles donne plutôt une paraphrase, mais elle me convient: "Le nombre n'est-il pas utilisé de façon pratique pour désigner la quantité et n'est-il pas en même temps une signification théorique pour désigner la qualité ». Mais qualitélquantité - cette opposition traditionnelle (un peu scolastique) me gêne ici; bien que je ne sache pas dire autrement en français. Il faudrait quelque chose comme le « combien ", le « quoi? ".

En tous cas je vois bien ce qui est indiqué: deux façons de s'y prendre, quand on fréquente les nombres. Or si cela était compris par les sages instruits au temps d'Evagre (en Orient) - en Occident c'est le temps de St Augustin - ce n'est plus le cas aujourd'hui ${ }^{14}$.

Résumons-nous. Pour Evagre (Guilbaud), le nombre 153 désigne d'abord une quantité, le nombre de poissons de la dernière pêche miraculeuse, mais aussi, et sans qu'il faille en faire une incidente, il possède une qualité propre, notamment d'être triangulaire, alors qu'il pourrait être carré comme 36 (qui se trouve être aussi triangulaire) ou même sphérique (ou rond) comme 25 (peut-être, parce que 25 se retrouve à l'identique dans toutes les puissances de 25 , ou dans celles de 5 , suivant ce théorème de Guilbaud, «le carré d'un nombre rond est encore plus rond »). 153 jouit ainsi d'une situation particulière dans le monde des nombres et de leurs relations. Il a une «signification théorique » ou une qualité arithmétique, chargée de l'expérience de générations de savants et de priants, que Dieu connaît en toute clarté.

Ceci n'est que le prologue, il faudrait maintenant pénétrer les 153 chapitres, les commentaires et les recherches qu'ils suscitent. Hélas, nous l'avons dit, le texte de Guilbaud est long et fourmille d'allusions de toute sorte et de vues originales. La paraphrase ou le résumé deviennent difficiles ou sans intérêt. Il faudrait tout éditer et ce

\footnotetext{
13 «Les œuvres du Très Haut vont par paires, en vis-à-vis », Ecclésiastique, 33, 14-15 et 42, 24. Aussi Ecclésiaste, 3, 1-8. De ce dernier texte Guilbaud appréciait fort la nouvelle traduction de Jacques Roubaud, Sous le soleil, vanité des vanités, Paris, Bayard, 2004.

${ }^{14}$ Cette dernière remarque s'applique en premier lieu aux exégètes, qui ne voient dans les «symboles numériques » des Pères du Désert et de la littérature médiévale, cistercienne en particulier, qu'une sorte de folklore primitif ou de superstition, un peu honteuse, un peu ridicule, qu'on doit ignorer ou traiter sur le mode ironique, pour ne pas risquer les moqueries des collègues. Et cela s'applique aux plus grands d'entre eux, Henri de Lubac notamment, dont Guilbaud cite «Une doctrine synthétique. 1. Symboles numériques », in Exégèse médiévale : Les quatre sens de l'Écriture, 2e partie, II, Paris, Aubier, 1964, p. 7-40. Cela s'applique aussi hélas aux mathématiciens qui n'aiment pas vraiment les mathématiques, ou aux alpinistes qui détestent la montagne. On n'est trahi que par les siens.

À propos des éditions Aubier, signalons incidemment que, d'après une note manuscrite des Ball.Trapp., Fernand Aubier était « un ami très cher » de Guilbaud. Cette amitié était « née dans un séjour d'une semaine à la trappe ».
} 
n'est pas le lieu. Et puis il s'agit d'évoquer Guilbaud. Evagre n'est qu'un prétexte, même si l'imaginer dans sa cellule du désert écrivant ses 153 articles a quelque chose de réjouissant. Alors oublions Evagre et reportons nous à la page « $\mathrm{M} »$ de 153 , une page « arithmétique », dans lequel action d'inventer et contemplation-compréhension dialoguent ensemble et conduisent à une réflexion sur la prière.

\begin{abstract}
J'enquêtais sur les attitudes de prière, et me voici derechef ramené vers l'exercice de la mathématique.

J'admirais Archimède; pas pour les historiettes répandues chez les doxographes, évidemment - mais parce que je lisais ses beaux textes mathématiques (André Weil, avec une emphase juvénile, célébrait le "sonore dialecte dorien ${ }^{15}$ ) - et il $m$ 'arrive de les lire encore, pour y contempler la naissance du calcul intégral, et la trace (mais morte) de cette force d'âme (ou d'esprit?)

Comment lire des écrits mathématiques?

La lecture, en ce cas, doit être activité. Chercher, par cette lecture, à mettre ses pas dans les pas d'un autre. Produire en soi-même des mouvements de pensée qui soient image de ceux que conduisait l'auteur - l'image ou l'écho, ou, plus simplement, la suite?

On s'est quelquefois préoccupé des découvertes, des inventions en arithmétique, géométrie et le reste. Mais comprendre est aussi mystérieux $q u$ 'inventer.

"Étonnants voyageurs ... " non pas «dites qu'avez-vous vu ... " ${ }^{16}$ (ce serait simple curiosité) mais : emmenez-moi! ou plutôt : laissez-moi partir. Car chacun sa voie.

Tout cela n'avait-il pas son analogue, si je voulais lire non pas «des prières 》-mais des écrits parlant de la prière.
\end{abstract}

Guilbaud note ensuite l'analogie qu'il y a entre cette lecture active et ce qu'en vocabulaire monastique, on appelle la lectio divina, une attitude qui consiste à se laisser interpeller et transformer par un texte sacré pris au hasard (ou pas tout à fait au hasard), par une seule phrase parfois, ou un seul mot, qui soudain se charge de transmettre l'expérience spirituelle de générations de croyants et de priants et qui, selon Grégoire le Grand, est une façon « de connaître le cœur de Dieu au travers des paroles de Dieu ». La lectio divina est une des observances cisterciennes, avec l'office divin et le travail manuel, vus comme autant de formes de prière, de pratiques priantes. Plusieurs grands spirituels ont tenté de préciser la nature de cette lectio divina (beaucoup plus en tout cas que de savants qui se sont essayés à l'analyse de la lecture arithmétique). Saint Ambroise la décompose en quatre moments, lectio, meditatio, oratio, contemplatio. Autant de «mots » difficiles à maîtriser dont Guilbaud montre les "glissements de sens » et les entremêlements à travers des textes de Guillaume de Saint-Thierry, Jean Gerson, Jean de la Croix, Thérèse d'Avila, Anselme Le Bail et Henri Brémond ${ }^{17}$. Occasion, une fois encore pour Guilbaud, d'expérimenter sa méthode, dérivée d'une sorte de nominalisme probabiliste. Les mots sont des choses, que l'on ne peut appréhender que statistiquement. Ce sont des objets probabilistes, comme les « tas de blé » de ses maîtres Borel et Darmois.

${ }^{15}$ N. Bourbaki, Eléments d'Histoire des mathématiques, nouvelle édition augmentée, Paris, Hermann, 1974, « Calcul infinitésimal », p. 207.

${ }^{16}$ Charles Baudelaire : «Le Voyage » III, (1859).

${ }^{17}$ Guilbaud nous dit qu'en 1932-1933, parallèlement au cours de Gilson, il lisait un classique cistercien, Dom Anselme Le Bail, L'Ordre de Cîteaux. « La Trappe », Paris, Letouzey et Ané, 1922, 1924, 1947.

Guilbaud cite, de Brémond, Histoire littéraire du sentiment religieux en France, 11 vol., Paris, Bloud et Gay, 1916-1933, nombreuses éditions successives, et aussi, L'inquiétude religieuse, Paris, Perrin, 1909, 1933. 
Suivant Brémond, Guilbaud compare en particulier la lectio divina (ou la lecture arithmétique) à la lecture "poétique », dans certaines circonstances, lorsque la raison et le cour se coalisent un court moment pour faire glisser le sens des mots. Mais ajoute-til : « il y a bien des façons de lire les poèmes. Et les poèmes sont divers » (page $V$ ), une autre façon de dire que la lectio divina ne saurait se définir autrement que par son nom, signe de ralliement d'une famille de sens multiples, parfois antagonistes.

Nous terminons, en reproduisant la dernière page du fragment 153 , la page Y, une page authentiquement guibaldienne :

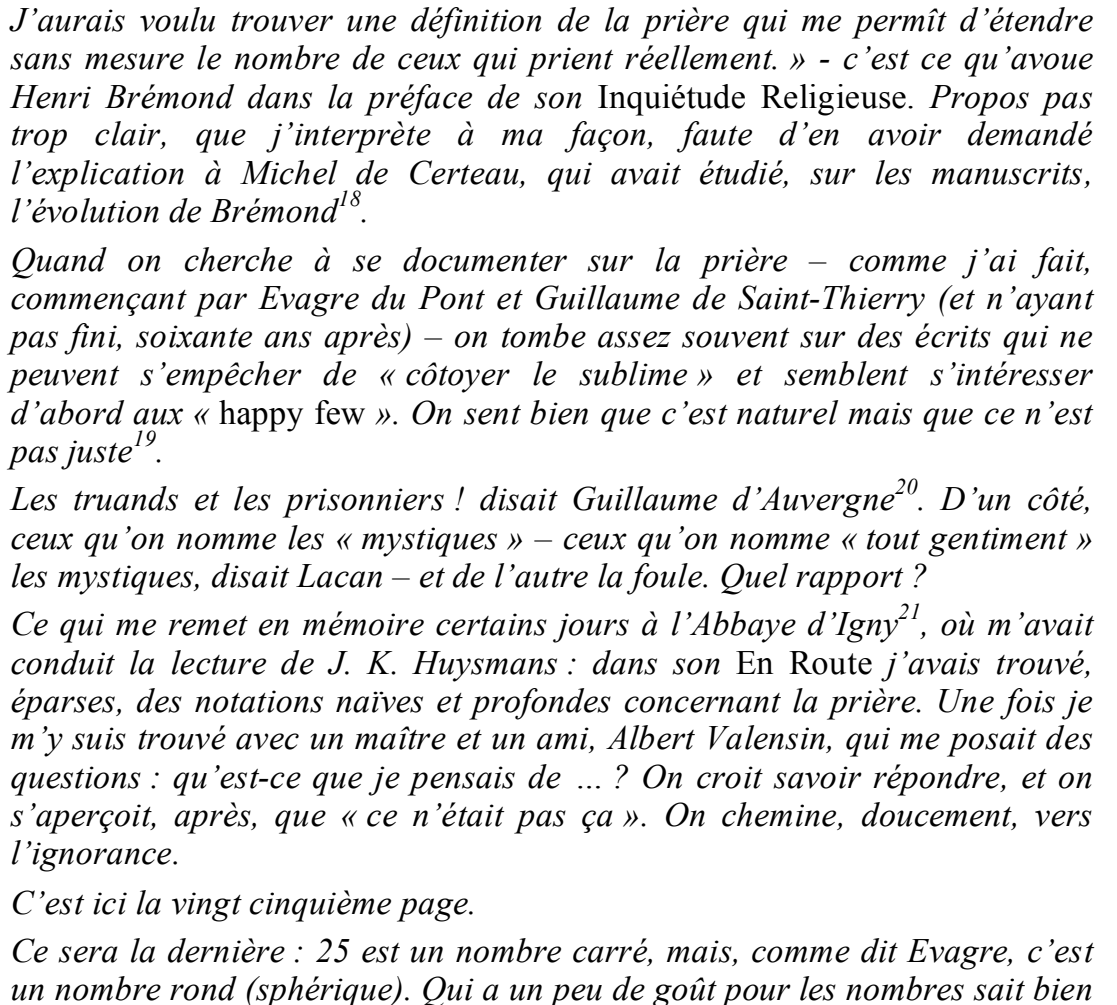
un nombre rond (sphérique). Qui a un peu de goût pour les nombres sait bien

\footnotetext{
18 Guilbaud cite un article paru en 1966 dans les Recherches de Sciences Religieuses, repris dans l'ouvrage, L'Absent de l'Histoire, Paris, Mame, 1973, 73-108.

${ }^{19}$ On sent bien que, par correspondance, Guilbaud pense aussi à l'arithmétique, et peut-être aussi à la montagne.

${ }^{20}$ Guilbaud précise (page T) cette allusion. Il s'agit d'un autre texte du recueil de Gerson, Initiation à la vie mystique, cité note (6) ; un texte au titre guibaldien, "La montagne de contemplation », p. 97, où Gerson parle du « très excellent docteur Guillaume, jadis évêque de Paris, de qui j'ai lu qu'il affirmait que les pauvres et les truands et les prisonniers lui avaient appris à prier ». Pierre Pascal donne la référence, notes 48, 49, 77-80. Gerson renvoie à Guillaume d'Auvergne, 1191-1249, qui a composé vers 1240, Rhetorica divina seu ars oratoria eloquentiae divinae, dont Gerson lui-même s'est beaucoup servi pour composer son Initiation. P. Pascal précise que le but de Guillaume est d'enseigner l'art et la pratique de la prière comme Aristote, Cicéron et Quintilien ont enseigné l'art de l'éloquence profane. Il cite à ce sujet Jérôme Lingenheim, L'art de prier. Essai sur la Rhétorique divine de Guillaume d'Auvergne, Lyon, 1934. La prière du truand est la plus simple possible, elle charge tous les saints du paradis d'intervenir pour lui, qui ne sait pas prier et qui n'a rien à donner, ou si peu !

${ }^{21}$ L'abbaye cistercienne Notre-Dame d'Igny a été fondée en 1128. Elle se trouve près de Reims. Fermée puis vendue sous la Révolution, elle a été rachetée en 1875 et rétablie en abbaye d'hommes en 1886. Celle-ci a été détruite pendant la guerre de 14. Rebâtie, elle est depuis 1927 une abbaye de moniales cisterciennes, OCSO. C'est en 1892 que Huysmans fit une retraite à la Trappe d'Igny, début de sa conversion, qu'il a racontée dans En route en 1895.
} 
que le carré d'un nombre rond est encore plus rond. Donc, pas de vingt sixième, ce serait encore la première (comme a dit le poète) ${ }^{22}$.

Il faut s'arrêter, toujours s'arrêter, s'arrêter sans arrêt. J'écrivais ces pages en prenant le temps (pas plus d'une ou deux par semaine) et sans destination (mais non sans destinataire). Pas de plan. Vagabondages, citations et questions - et souvenirs de rencontres. (quelques noms d'amis vénérés : mon petit Panthéon).

Tout ça pour relire plus tard. Inch Allah.

\section{IN OMNIBUS REQUIEM QUAESIVI}

Nous avons déjà été trop long. Le plan initial prévoyait un arrêt sur le fragment Lumen in manibus, une ballade sur le thème des intermittences, ou, si on préfère, sur la connaissance certaine. Mais nous devons abréger, et nous ne pouvions finir sans évoquer les bribes du dernier fragment des Ball. Trapp. Des textes disparates, manuscrits, qui sont arrivés en ordre dispersé par la poste jusqu'à la veille de sa mort. Ces morceaux d'un fragment d'un manuscrit potentiel s'intitulent, In omnibus requiem quaesivi, un autre slogan de Guilbaud, qu'il citait volontiers et qu'il nous faut présenter rapidement ici, en paraphrasant sa recherche.

La traduction en français, cette fois-ci, semble ne poser aucun problème : « en toutes choses, j'ai cherché la paix », mais le sens en est-il plus clair pour autant? Chacun peut y mettre ce qu'il veut. Est-ce une citation biblique ? Oui et non ? Comme toujours, un mélange des deux, ou un glissement. Dans la traduction latine de la Vulgate, on lit , Ecclésiastique 24, 11 : «et omnium excellentium et humilium corda virtute calcavi et in his omnibus requiem quaesivi et in haereditate ejus morabor». Rappelons que c'est la «Sagesse » qui parle et qui fait son propre éloge. Voyons les traductions françaises. Par exemple, la Bible des moines bénédictins de Maredsous, qui traduit, presque mot à mot : «J'ai eu sous mes pieds par ma puissance, les cœurs de tous les hommes, grands et petits ; parmi toutes ces choses j'ai cherché un lieu de repos, et j'habiterai dans le domaine du Seigneur», tandis que la Bible de Jérusalem, qui s'éloigne de la Vulgate, pour se rapprocher des versions grecque et hébraïque (partielle) du Siracide ${ }^{23}$, traduit : c'est ainsi « que dans la Cité bien-aimée j'ai trouvé mon repos, qu'en Jérusalem j'exerce mon pouvoir ».

Chercher (de la Vulgate) ou trouver (de la Septante) ? Voilà un flou propice aux glissements de sens jusqu'au slogan de Guilbaud, qui supprime le « his » de « his omnibus », ce qui simplifie un peu la chose. Voyons d'abord quelques intermédiaires, avant de tenter de nous approcher du sens véritablement guilbaldien du slogan, sens qui d'ailleurs, par définition, ne saurait être le bon.

D'abord une fausse piste, ou plutôt un emprunt biblique détourné qui ne nous intéresse pas. Si on tape « in omnibus requiem » sur Google, on tombe inévitablement sur un proverbe, assez rudimentaire, répété à satiété depuis le $\mathrm{XV}^{\mathrm{e}}$ siècle, jusqu'à Anatole France et les autres : "In omnibus requiem quaesivi, et nusquam inveni nisi in angulo cum libro », « Partout j'ai cherché la paix, et je ne l'ai trouvé nulle part qu'avec un livre dans un coin », qui est attribué sans preuve à Thomas a Kempis par un grand

\footnotetext{
${ }^{22}$ Gérard de Nerval, «Artémis », sonnet extrait des Chimères (1854) : «La treizième revient... C'est encore la première ».

${ }^{23}$ L'établissement du texte ou des textes du Siracide présente des difficultés qui ne semblent pas prêtes d'être surmontées, on verra par exemple la thèse de Thierry Legrand, « Le Siracide : problèmes textuels et théologiques de la recension longue », Faculté de Théologie protestante, Strasbourg, 1996.
} 
nombre d'auteurs qui se recopient les uns les autres, jusqu'à Umberto Eco, sans trop y croire. Mais on est loin du compte, un livre ne donne jamais la paix ou si peu ! Il peut divertir, être source d'information ou d'interrogation, peut-être aussi le point de départ d'une lecture poétique ou arithmétique ou divine, mais alors il n'est qu'un signe; le coeur intervient et les pratiques mystiques, et les exercices mathématiques, et l'alpinisme, et tant d'autres choses.

La seconde piste est plus intéressante. On trouve le slogan In omnibus, dans une des Pensées éparses de Pascal, La 889, Br 165: «Pensées. In omnibus requiem quaesivi. Si notre condition était véritablement heureuse, il ne nous faudrait pas divertir d'y penser pour nous rendre heureux ». «Pensées » à laquelle on ajoute La 890, Br 436 bis (d'actualité) : " Toutes les occupations des hommes sont à avoir du bien et ils n'ont ni titre pour le posséder justement, ni force pour le posséder sûrement. De même la science, les plaisirs : nous n'avons ni le vrai ni le bien », ainsi que le fragment placé dans la liasse «Misère », La 70, Br 165 bis, qui recopie une partie du précédent : « Si notre condition était véritablement heureuse il ne faudrait pas nous divertir d'y penser ». On est là sur un terrain plus solide avec des siècles de commentaires érudits pour nous éclairer. Tous ceux-ci, sans exception, signalent en note que la citation latine de La 889 est extraite de Ecclésiastique 24, 11. Suivent éventuellement des développements sur le divertissement et la paix chez Pascal, la citation biblique étant détournée de son « sens biblique », comme toujours, pour affirmer davantage encore la faiblesse de la condition de l'homme qui ne saurait trouver jamais la paix (même pas dans un livre). Mais si Pascal avait voulu citer la Vulgate, n'aurait-il pas écrit In his omnibus... ? Pourquoi ne l'a-t-il pas fait? Certainement pas pour évoquer le proverbe in angulo cum libro. Sans doute avait-il autre chose en tête ? Il faut chercher plus loin. Nous donnons ci-dessous la solution des Ball. Trapp., qui résoud simultanément l'énigme La 889 et le (second) slogan de Guilbaud.

Commençons par regarder le manuscrit autographe, de la main de Pascal, que Guilbaud a décalqué et joint au dossier. Suivons le :

« Nous avons deux papiers autographes

1) Le premier est divisé par des traits (de la main de Pascal)

- ce qui fait trois morceaux,

- il a été divisé en deux et non en trois par Lafuma $\left(n^{\circ} 889,890\right)$ qui n'a tenu compte que de la $2^{\mathrm{e}}$ séparation,

- (pourquoi ?) sans doute : séparer en deux numéros («Si notre condition était ...», «Toutes les occupations ... ») lui semble nécessaire parce que « Si notre condition... » a été recopiée (de la main même de Pascal),

- recopiée (et non pas découpée).

2) Pour être classée dans la liasse « Misère ».

C'est le second papier (Lafuma lui donne le numéro 70)

Remarque : Brunschvicq avait noté ce doublet.

Dans certaines éditions il y a ${ }^{\circ} 70$ et 70 bis.

Pour quelqu'un sans préjugés, il me semble qu'il verrait 4 morceaux :

1) Le titre obscur : Pensées

(on le trouve en tête plusieurs fois)

2) La citation latine 
3) Si notre condition...

4) Toutes les occupations...

Quels liens peut-on trouver entre ces morceaux?

À mon avis : aucun, sinon d'avoir été écrits sur le même papier.

Et sans doute le même jour, ou à peu de distance ».

Donc, selon Guilbaud la citation latine n'est pas là pour introduire ou renforcer la suite, c'est autre chose, mais quoi ?

Oublions un moment Pascal et revenons à Guilbaud.

Pour Guilbaud, le slogan In omnibus est, comme Ad quid venisti un souvenir cistercien, et plus précisément « un souvenir de mes premiers longs séjours à Port-duSalut : ces capitula répétés, plusieurs fois par jour, dans les Petites Heures, m'étaient restés en mémoire (Souvenirs « liturgiques », voir ce qu'en disait J. K. Huysmans ${ }^{24}$ ) ». Plus précisément, l'Office divin, avant les aménagements pré et postconciliaires, était doublé, du «Petit office de la Très Sainte Vierge », un office liturgique remontant peutêtre à l'Église primitive, en tout cas attesté dès le VIII ${ }^{\mathrm{e}}$ siècle. La Règle de saint Benoît ne prescrit rien à ce sujet, mais il semble que l'office de la Vierge était dit dans les monastères dès le $\mathrm{IX}^{\mathrm{e}}$ siècle. Lors de la prédication de la première croisade, le pape Urbain II ordonna (par mesure de précaution) que l'office de la Vierge soit dit obligatoirement par tous ceux qui étaient tenus à l'office canonial. Les abbayes cisterciennes gardèrent cet usage jusque tard dans les années 1950. Ce qui explique que Guilbaud, lors de ses séjours trappistes, l'ait entendu aux Matines et lors des sept offices du jour. L'office de la Vierge est une longue méditation sur le mystère de Marie, l'Annonciation, la Visitation, etc, bâtie, comme l'office ordinaire sur des hymnes, des psaumes et des lectures (les leçons ou les capitules) tirées de la Bible, mais transposées au mystère de la Vierge, assimilée à la Sagesse de Dieu, glissement de sens, allégorie poétique, la «Nouvelle Eve» devenant Verbe. La première leçon, tirée de l'Ecclésiastique 24, 11-20, commence ainsi «In omnibus requiem quaesivi et ....», qu'il faut traduire : Marie a cherché le repos en se conformant à la volonté de Dieu etc. ${ }^{25}$. Cette leçon est chantée en grégorien par un choriste seul. Elle a été intégrée à d'autres liturgies de la Vierge, plus mondaines et plus ornées, par exemple les Vêpres de la Vierge de Monteverdi. Pour un retraitant, qui se pose la question Ad quid venisti ?, ce peut être une façon de trouver la paix: In omnibus..., sans his, puisque le texte commence au milieu du verset, le privant de son antécédent.

Mais que vient faire Pascal dans cette affaire? Pour Guilbaud, c'est assez évident : «Blaise Pascal a fait plusieurs « retraites » à Port-Royal des Champs. Il y a suivi les offices. Et sa sœur Gilberte, en écrivant la vie de son frère, dit : «Il avait un amour sensible pour tout l'office, mais surtout les Petites Heures». On peut donc comprendre La 889 de la façon suivante: In omnibus requiem quaesivi est un «souvenir liturgique» et non biblique, sans rapport avec la suite. Plus qu'une confirmation de la faiblesse de la condition humaine, ce serait plutôt une affirmation de

\footnotetext{
${ }^{24}$ Cf. note 21 supra.

${ }^{25}$ Le texte du Siracide 24 semble prophétiser le mystère de la Vierge-mère. Au verset 12, la Vulgate écrit : «Tunc praecepit, et dixit mihi Creator omnium : et qui creavit me, requievit in tabernaculo meo. » (Alors le Créateur de toutes choses m'a commandé et m'a dit : et lui qui m'a créée a reposé dans mon tabernacle), ce qui conforte la probabilité d'une lecture mariale du texte, d'ailleurs rejeté comme apocryphe par les traditions juives (non alexandrines) et protestantes.
} 
sa dignité. Sans chercher à pénétrer plus avant, on peut au moins imaginer que Pascal avait trouvé la paix à Port-Royal, parfois ${ }^{26}$. requiem ...».

À la veille de sa mort, Guilbaud écrivait : «pas de plaintes : in omnibus

\section{AD QUID VENISTI ?}

Au terme de ce survol approximatif des ballades trappistes, il convient de revenir à la question initiale, Ad quid venisti ?, pour conclure avec Guilbaud :

«Si je suis venu

C'est justement pour pouvoir me poser la question :

Pourquoi es-tu venu?

Ça ressemble à une plaisanterie, et c'est en même temps un vrai raisonnement (« récursif »).

Saint Anselme, Pascal, Kierkegaard, et d'autres, ont construit certaines de leurs exhortations sur de semblables paradoxes. Aussi les premiers cisterciens qui ont commenté les phrases (mystérieuses) du Cantique des cantiques $1,6^{27}$ :

Si ignoras te ... egredere ...

Si tu ne te connais pas ... sors ...

Mais évitons l'enflure et reprenons les sentiers piétons ».

\footnotetext{
${ }^{26}$ Guilbaud avait noté un certain nombre de références cisterciennes chez Pascal, mais il n'a pu achever cet ultime fragment des Ball. Trapp.

${ }^{27}$ Le Cantique des Cantiques est également très présent dans l'Office de la Vierge, et surtout dans la théologie mystique cistercienne, l'épouse pouvant être vue successivement ou simultanément comme une allégorie de la Vierge Marie, du Christ et de l'Église, entremêlements de sens, ou cohabitation inévitable. Bernard de Clairvaux avait entrepris de commenter le Cantique, verset par verset pour, l'édification de ses moines : 86 sermons interrompus par sa mort en 1153. Le commentaire fut repris par Gilbert de Hoyland, abbé de Swineshead (Lincolnshire), qui composa 47 autres sermons interrompus aux versets 810 du chapitre 5, par sa mort vers 1172 . L'entreprise devait finalement être menée à son terme par Jean, abbé de Ford (Dorset), qui composa les 120 derniers sermons au début du XIII ${ }^{\mathrm{e}}$ siècle (John Ford, comme l'appelait Guilbaud, est mort vers 1214). Signalons que les 120 sermons de Jean de Ford, comme ceux de Gilbert de Hoyland, ont été traduits en français et annotés par Pierre-Yves Emery, frère de Taizé, et publiés par l'abbaye Notre Dame du Lac au Canada, dans la Collection Pains de Cîteaux (n ${ }^{\circ}$ 6, 7, 15, 16, 17, 1994 - 2001), une collection fondée en 1959, par le père Robert Thomas de l'abbaye de Sept-Fons, un ami de Guilbaud, qui, au reste, avait participé «incognito » à cette traduction. Quant aux sermons de Bernard, ils sont traduits et annotés dans la collection Sources chrétiennes $\left(\mathrm{n}^{\circ} 414,431,452,472,511\right.$, 1996-2007).
} 
70

B. BRU

$\log \stackrel{\text { bit }}{=}$

Ball.Trapp:

- Mantrecats

- Belval

Chiprey - Rochefort

Igng

- Corveman

$*$ Q nod

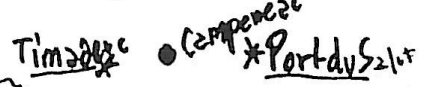

* Citeon

* Belleterarám

Sertons

* Dombes

* Tanié

13 * veritalles séjoury (plosisos ruil centecative

Chinderian? s deaque fais)

nergeso * Aiguebdle

8 - simply vitite daus la journie (unatfic)

Dicert

Penjees

12888

12890

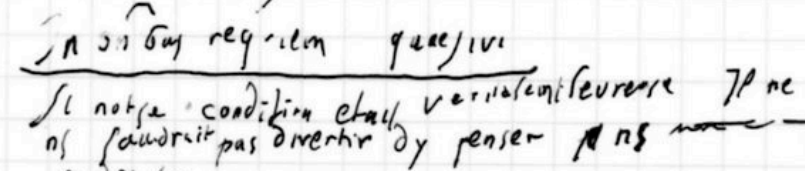
resore hevreus

Toura les ocupotion de homme sont a a var do lien etlls nout al titre pas possecor justement In pla pojsevie surement Demenee la science esplasion n'avas ni le vray ni le bien

1270 now Dirctir $\partial / p^{\text {endu-1 }}$ 